\title{
A clarion call to introduce artificial intelligence (Al) in postgraduate medical physics curriculum
}

\author{
Kwan Hoong $\mathrm{Ng}^{1}$. Jeannie Hsiu Ding Wong ${ }^{1}$
}

Published online: 10 January 2022

(c) Australasian College of Physical Scientists and Engineers in Medicine 2022

Artificial intelligence (AI) is expanding extremely rapidly in almost every sphere of human activity, and there is no exception in medicine. Medical physicists are healthcare professionals intricately involved in many aspects of patient care. The ACPSEM position statement on the role of medical physicists also states that the profession should be "engaging with opportunities offered by AI and machine learning as well as linking and developing datasets to inform clinical practice" [1]. However, are we well equipped with the necessary AI knowledge and skills to accomplish this? Is the state-of-the-art medical physics education and training programme preparing the younger generation to contribute meaningfully and assume leadership roles in implementing $\mathrm{AI}$ in healthcare?

In the last decade or so, computing and technological advancement have led to the exponential growth of research in AI. Many AI-themed medical journals have sprung up, such as Radiology: Artificial Intelligence and Journal of Medical Artificial Intelligence. The number of medical AI studies by year from 2010 to 2020 ; and by medical specialties indicates an exponential increase, notably in pathology, radiology, surgery, psychiatry, oncology, and neurology; all of these medical physics have been actively contributing [2]. Using the search terms of "machine learning" OR "deep learning" in the PubMed database resulted in an astounding 90,156 articles published since 2010 and 31,103 publications in 2021 alone!

A recent global survey on the perception of medical physicists towards the relevance and impact of AI revealed that more than $85 \%$ of the respondents agreed that AI would play a prominent role in our practice [3]. However, $40 \%$ of the respondents learned AI on their own, and $40 \%$ of them reported do not have AI skills. The survey clearly showed

Kwan Hoong Ng

ngkh@ummc.edu.my

1 Department of Biomedical Imaging, Faculty of Medicine, Universiti Malaya, Kuala Lumpur, Malaysia that the medical physics community is aware of the impending and evolving profession, and the general view was that AI should be part of the medical physics curriculum. A similar Europe-focused survey also reported the same findings [4].

Recognising the importance of getting started, the ASEAN College of Medical Physics organised a short hands-on course on AI during the recent 19th South-East Asian Congress of Medical Physics (SEACOMP) [5]. The Google Colab was used to introduce participants to coding with clinical examples. We encourage more of such initiatives to be started to promote learning.

However, learning AI is not achievable in short two-day ad-hoc courses; a well-structured curriculum is necessary. Medical physicists must understand the basics of AI to contribute meaningfully in various medical fields, evaluate AI-based studies and clinical validation, and understand the limitations and opportunities AI provides. We encourage medical physics educators to develop a strategy to incorporate AI module in the curriculum. Towards that end, we propose that the (1) postgraduate medical physics programme should include a more comprehensive and wellstructured AI module, (2) extending the medical physics education and training time and (3) inclusion of appropriate computer/data science experts in the curriculum delivery.

We have consulted various syllabi published by AAPM, IPEM, IAEA-currently, there is no specific module of AI.in the curriculum. Even the latest IAEA training course series (TCS)-56 (revised)—Postgraduate Medical Physics Academic Programmes also does not include a more extensive AI module in the curriculum [6]. Most postgraduate medical physics programmes comprise one to two years of education, typically not including a specific AI module [7]. In addition, the staffing of a standard medical physics programme may not always include an $\mathrm{AI} /$ computer science background faculty.

In 2021, EFOMP developed a curricular programme on AI [8]. The proposed AI curriculum is very extensive 
and detailed, comprising of a basic and advanced module. Implementation of the complete module would undoubtedly demand a minimum of one year. Thus, incorporating the said curriculum in the current standard one to two-year medical physics curriculum would be challenging unless one considers extending the education and training period. Nevertheless, we believe that this should be given serious consideration.

We should consider actively including computer/data scientists in the AI training of medical physicists. Without the proper training, the chances of the research translating to actual clinical practice will be minimal, and the opportunity of a medical physicist making a significant impact will be missed. The worse case scenario is that medical physicists may be excluded from the inter-discipline collaboration entirely; thus we should prioritise integrating these skills as part of the medical physics skillset.

Now is the opportune time to implement AI in our postgraduate medical physics curriculum. A wait-and-see attitude will only jeopardise our traditionally well-respected leadership in physics and engineering applications in medicine.

There is no room for complacency. Let us make a difference.

\section{References}

1. ACPSEM. ACPSEM position statement. The role of physicists, scientists and engineers in medicine in Australasia. 2018. https:// www.acpsem.org.au/documents/item/18
2. Meskó B, Görög M (2020) A short guide for medical professionals in the era of artificial intelligence. NPJ Digital Med 3(1):126

3. Santos JC, Wong JHD, Pallath V, Ng KH (2021) The perceptions of medical physicists towards relevance and impact of artificial intelligence. Phys Eng Sci Med 44(3):833-841. https://doi.org/10. 1007/s13246-021-01036-9

4. Diaz O, Guidi G, Ivashchenko O, Colgan N, Zanca F (2021) Artificial intelligence in the medical physics community: an international survey. Physica Med 81:141-146

5. Ng KH, Saw SN, Loo CK (2021) Equipping medical physicists with artificial intelligence knowledge and coding skills. Med Phys Int. http://mpijournal.org/pdf/2021-02/MPI-2021-02.pdf

6. IAEA (2021) Postgraduate medical physics academic programmes. International Atomic Energy Agency, Vienna. https:// www-pub.iaea.org/MTCD/publications/PDF/TCS-56_(Rev.1) web.pdf

7. Wong JHD, Ng KH, Sarasanandarajah S (2019) Survey of postgraduate medical physics programmes in the Asia-Oceania region. Physica Med 66:21-28

8. Zanca F, Hernandez-Giron I, Avanzo M, Guidi G, Crijns W, Diaz O, Kagadis GC, Rampado O, Lønne PI, Ken S, Colgan N, Zaidi H, Zakaria GA, Kortesniemi M (2021) Expanding the medical physicist curricular and professional programme to include artificial intelligence. Phys Med 83:174-183

Publisher's Note Springer Nature remains neutral with regard to jurisdictional claims in published maps and institutional affiliations. 